\title{
Prehension Deficits in Amblyopia
}

\author{
Simon Grant, Dean R. Melmoth, Michael J. Morgan, and Alison L. Finlay
}

Purpose. Visual defects associated with amblyopia have been extensively studied, but their impact on the performance of everyday visuomotor tasks is unclear. This study evaluates eye-hand coordination (prehension) skills in adult amblyopes compared with normal subjects.

MeTHods. Twenty amblyopes (10 strabismic, 10 nonstrabismic) with different degrees of visual acuity loss (mild, moderate, or severe) and stereodeficiency (reduced or undetectable) participated, along with 20 matched control subjects. Subjects reached, precision grasped, and lifted cylindrical household objects (two sizes, four locations) using binocular vision or just the dominant or amblyopic (nondominant) eye, while the actions of the preferred hand were recorded. Various indices of prehension planning and online control were quantified for all trials $(n=48)$ performed under each viewing condition.

Results. Initial reaching behavior and grip shaping before object contact, which result from movement programming, were relatively normal in the amblyopic subjects, despite their vision losses. By contrast, they exhibited a range of deficits under both binocular and nondominant eye conditions in their final approach to the object (terminal reach) and when closing and applying a grasp. These impairments included prolonged execution times and more errors compared with control subjects, the extents of which covaried with the existing depth of amblyopia, although not with its underlying cause.

Conclusions. Visuomotor adaptations in amblyopes are relatively minor and limited to aspects of movement planning. Their deficits in movement execution should benefit, however, from treatments that restore spatial acuity and binocularity to progressively normal levels and so deserve more explicit consideration when assessing therapeutic outcomes. (Invest Ophthalmol Vis Sci. 2007;48:1139-1148) DOI:10.1167/iovs.060976

mblyopia is a common developmental disorder of spatial
vision with an estimated prevalence, depending on diag-
nostic criteria, of $\sim 1.5 \%$ to $3 \%{ }^{1-3}$ The main associated risk
factors are strabismus (ocular misalignment), anisometropia
(significant refractive imbalance), and visual deprivation (cata-
ract, corneal lesions) before the age of 7 years, ${ }^{4,5}$ most often
affecting one eye. It is characterized by loss of visual acuity
(VA), contrast, and position sensitivity in the central field of the
affected (deviating, ametropic, or deprived) eye and by re-
duced binocularity and stereoacuity (SA). The severity of these

From the Department of Optometry and Visual Science, The Henry Wellcome Laboratories for Visual Sciences, City University, London, United Kingdom.

Supported by The Wellcome Trust, Grant 066282

Submitted for publication August 17, 2006; revised October 9 and November 9, 2006; accepted January 24, 2007.

Disclosure: S. Grant, None; D.R. Melmoth , None; M.J. Morgan, None; A.L. Finlay, None

The publication costs of this article were defrayed in part by page charge payment. This article must therefore be marked "advertisement" in accordance with 18 U.S.C. $\$ 1734$ solely to indicate this fact.

Corresponding author: Simon Grant, Department of Optometry \& Visual Science, The Henry Wellcome Laboratories for Visual Sciences, City University, Northampton Square, London EC1V OHB, UK; s.grant@city.ac.uk. defects and the extent to which peripheral vision is also involved depend on several variables, including its etiology. Persons with strabismus, for example, tend to have more profound central deficits with relative sparing of peripheral functions, than do nonstrabismics. ${ }^{6-9}$ There is no overt disease. Primate models of the condition, ${ }^{10-12}$ supported by recent human neuroimaging studies, ${ }^{13-15}$ have repeatedly shown that the defects result from abnormal development of responses to the affected eye in the primary visual cortex (area V1) and at higher cortical levels, where distinct dysfunctions underlying strabismic versus nonstrabismic amblyopia most likely arise. ${ }^{16}$

Major clinical resources are expended in evaluating and ameliorating the vision losses in amblyopic patients, with the predominant treatment modality involving lengthy patching (occlusion) of the fellow eye. Early detection and intervention are believed to be important for good recovery, as the deficits become less tractable with age and declining cortical plasticity. ${ }^{5}$ Restoration of good VA in the amblyopic eye is an accepted benchmark of success, although recovery of highgrade binocularity is less prioritized, partly because occlusion therapy does not promote visual cooperation between the two eyes. A recent systematic cost-benefit review, ${ }^{17}$ however, has challenged the validity of amblyopia treatment. The primary grounds for this position were a lack of objective evidence that amblyopes are functionally impaired in their daily activities, allied to the suggestion that "people with only one good eye since childhood [may] develop compensatory visual mechanisms" 17 that render therapy unnecessary. Although somewhat partial in dismissing extant evidence that reading ability can be impaired in amblyopia ${ }^{18}$ (and see Ref. 19), the review by Snowdon and Stewart-Brown ${ }^{17}$ has nonetheless highlighted the lack of data about the impact of the disorder on the control of visually guided actions.

Reaching for and grasping objects (prehension) are highly skilled everyday activities that are partly mediated by higher "dorsal stream" (posterior parietal) cortical areas, ${ }^{20}$ the speed and accuracy of which normally depend on aspects of spatial vision that are defective in amblyopia. They are also readily amenable to investigation, as their kinematics can be noninvasively recorded and quantified. Proficient reaching transports the hand quickly to the intended target and requires accurate information about its spatial location. Precision grasping is a more complex action. It begins with a maximum anticipatory opening of the hand (termed preshaping) while it is decelerating close to the goal object, followed by rapid closure of the digits onto the most stable-looking grasp points, the skilled performance of which requires visual computation of the target's intrinsic three dimensional (3-D) properties (e.g., shape and size) and depth relative to the approaching hand. Kinematic studies in normal adults have shown that reaching becomes impaired under conditions of restricted central or peripheral vision ${ }^{21,22}$ and when either monocular or binocular distance cues are altered, ${ }^{23,24}$ suggesting that many sources of spatial information can contribute to guiding hand transport. In contrast, the control of grasping appears to be particularly dependent on binocular vision, since grip formation and application are disrupted by temporarily occluding one eye $\mathrm{e}^{25-27}$ or the central fields of both eyes ${ }^{21}$ and when disparity cues to object shape and hand-target depth are compromised. ${ }^{28,29}$ 
Moreover, these eye-hand coordination skills are normally acquired over a protracted period of development extending through infancy, beyond the critical period for amblyopia, until around 12 years of age. ${ }^{30-34}$

These considerations suggest that prehension is adversely affected in amblyopia. An alternate possibility is that individuals with this condition could achieve proficiency through prolonged reliance on monocular information provided by the "good" eye. However, although supranormal spatial vision (e.g., contrast and positional sensitivity) has been found in the dominant (DOM) eye of some adult anisometropes, vision in this eye of strabismics is typically reported to be worse than normal. ${ }^{8,35-37}$ Whether visuomotor adaptations occur in amblyopia may thus depend on its underlying cause, as well as its severity. We have examined these issues by comparing the prehension skills of 20 adult amblyopes with those of normally sighted subjects when using their DOM eye, amblyopic/nondominant (N-D) eye, or both eyes together to plan and execute their movements. The amblyopes consisted of an equal number of strabismic and nonstrabismic individuals, each with a range of VA and SA losses. We found little evidence of enhanced DOM eye performance in the patients compared with control subjects, but there was a range of reaching and grasping deficits in binocular and amblyopic eye conditions that generally correlated with the extent of their visual impairments.

\section{Materials AND Methods}

\section{Participants}

The study involved 20 adult amblyopes (aged 18-48 years) and 20 normal subjects with no history of neurologic or ocular disorder (other than refractive error) who were individually matched to the patients by age, gender, and preferred hand. All participants underwent a full clinical visual assessment and completed a handedness questionnaire (Edinburgh Inventory ${ }^{38}$ ), before the prehension experiment. The visual examination included refraction, tests of crowded letter (logarithm of the minimum angle of resolution; logMAR) VA and contrast sensitivity (Pelli-Robson chart) applied in sequence to the N-D eye, DOM eye, and binocularly. In the normal subjects, the DOM eye was that used for sighting, at near. Binocular vision was further evaluated from measures of sensory (Bagoloni lenses, Worth's lights) and motor fusion (variable prism bar) and of their crossed and uncrossed SA thresholds (Randot-E and/or TNO tests). Key clinical details of each patient are summarized in Table 1. A diagnosis of anisometropia (with or without an accompanying strabismus) required a difference of at least $1 \mathrm{D}$ between the two eyes in the corresponding major meridians. The existing depth of amblyopia in the affected eye of each patient was categorized as "mild" $(n=7)$, "moderate" $(n=9)$, or "severe" $(n=$ $4),{ }^{2}$ depending on whether its VA fell with the ranges 0.2 to $0.49,0.5$ to 1.0 , or $>1.0$, respectively (approximate Snellen equivalents: $6 / 12$ to $6 / 18,6 / 18$ to $6 / 60$, and worse than $6 / 60$ ). Average binocular, DOM and $\mathrm{N}-\mathrm{D}$ eye $\log \mathrm{MAR}$ acuities in the normal adults were $-0.16,-0.11$, and -0.08 , respectively. Most of the patients had received occlusion therapy before age 10 . The exceptions were subject NS3 for whom occlusion was prescribed but not complied with and subjects NS9 and NS10 who never sought treatment. The study was approved by the Senate Research and Ethics Committee of City University. All protocols accorded to the guidelines of the Declaration of Helsinki.

\section{Prehension Kinematics}

The procedures adopted were identical with those detailed by Melmoth and Grant. ${ }^{27}$ In brief, subjects sat at a table in a well-lit laboratory environment, initially gripping a circular "start" button between the thumb and index finger of the preferred hand, the button being situated $120 \mathrm{~mm}$ from the near table edge along the subject's midline body axis. Subjects wore liquid crystal goggles that were opaque at rest, but opened to signal the start of each trial, and closed again 3 seconds later. These fitted comfortably over any corrective lenses that the subjects usually wore and were accustomed to. Two of the anisometropes (NS4, NS8) possessed suboptimal prescriptions (which they did not wear continuously), and cited "visual discomfort" from full correction. In both cases, best correction slightly improved their $\mathrm{N}-\mathrm{D}$ eyes' VA as given in Table 1 , but did not change the amblyopia category to which they were assigned. The existing prescriptions were not updated in these cases, to avoid introducing a sudden and unpleasant change in their vision just before the prehension experiment.

Subjects planned and executed their movements under three viewing conditions, which were randomized from trial-to-trial: DOM eye only, N-D eye only, or with binocular vision available. They were instructed to use a precision grip to pick up the object on the table employing a natural, swift, and accurate movement; put it down to one side; and return the hand to the starting position. Practice trials (two to four for each view) were given to ensure that these instructions were followed appropriately. Targets were brightly colored, spatially detailed cylindrical household objects of either small $(24 \mathrm{~mm})$ or large (48 mm) diameter, placed near $(200 \mathrm{~mm})$ or far $(400 \mathrm{~mm})$ at $10^{\circ}$ from the start position in either ipsi- or contrahemispace relative to the reaching limb. Target presentation was also randomized from trial-totrial, to reduce repetitive movements. This and the other procedures used were intended to mimic real-world conditions as closely as possible. That is, the environment always contained a variety of monocular (pictorial) distance (e.g., perspective, height-in-scene) and depth (e.g., surface texture) cues, and subjects were free to move their heads, generating motion parallax and/or optic flow, that they could exploit when performing the task.

To record the participant's movements, the 3-D spatial coordinates of lightweight infrared (IR) reflecting markers attached to the wrist and to the nails of the thumb and index finger of the preferred hand were captured by three IR-emitting and detecting cameras (Proflex; Qualisys $\mathrm{AB}$, Gothenburg, Sweden) triangulating the workspace from above. Other IR markers were fixed to the upper surface of the goal objects. The recording rate was $60 \mathrm{~Hz}$, with a spatial resolution of $<0.5 \mathrm{~mm}$. Recording onset and termination were synchronized with opening and closure of the liquid crystal goggles. Subjects performed the 24 (3 view $\times 4$ location $\times 2$ object size) trials in six separate blocks, in the same random order, with brief rest periods between.

Thirteen dependent measures of each movement's kinematics were quantified (see Table 2 for details). Two were general ones: the reaction time (RT) from goggle opening to movement onset (MO) at the start of the reach, indicating the planning plus initiation time; and the total movement time (MT), from MO to the end of the movement (ME)-an index of the efficiency of execution. Other measures were related to the reach (analyzed mainly from the wrist marker) or the grasp (analyzed mainly from the markers on the digits) or to coupling between the two movement components. For these, the moment of initial object contact (OC), when the target was first displaced by $\geq 1$ $\mathrm{mm}$ from its original position was used as a key transitional landmark between the two. There were four dynamic measures of the reaching kinematics: its peak velocity (PV) and the total reach duration (RD), with this latter broken down into consecutive early and late segments, representing the time from $\mathrm{MO}$ to peak deceleration (ttPD) and the low-velocity phase (LVP) of the final approach. Grasp dynamics was similarly broken down into early-to-late segments, comprising the time to peak grip (ttPG) at preshaping, the grip closure time (GCT) from peak grip to $\mathrm{OC}$ and the grip application time (GAT), during which the object was manipulated before being lifting. Three further measures were related to spatial aspects of the grasp: the width of the peak grip aperture (PGA), its distance from the object (dPG) at pre-shaping, and the width of the grip at object contact (GOC). The final measure of how well the reach and grasp were coordinated (R-GC), was the time between the digit's initially contacting the object and the minimum wrist velocity (see Fig. 1). The rationale behind these was that early measures of the reach (i.e., PV, ttPD) and grasp (e.g., ttPG, PGA) are known to be programmed on the basis of target distance and size 
TABLE 1. Details of the Amblyopic Subjects

\begin{tabular}{|c|c|c|c|c|c|c|c|c|}
\hline \multirow[b]{2}{*}{ Patient } & \multirow{2}{*}{$\begin{array}{c}\text { Sex, } \\
\text { Age } \\
\text { (y) }\end{array}$} & \multicolumn{3}{|c|}{ logMAR Visual Acuity } & \multirow[b]{2}{*}{ Prescription } & \multirow{2}{*}{$\begin{array}{c}\text { Binocularity } \\
\text { Bagolini, Xed SA }\end{array}$} & \multirow[b]{2}{*}{ Observations } & \multirow{2}{*}{$\begin{array}{l}\text { Depth of } \\
\text { Amblyopla }\end{array}$} \\
\hline & & BO & DOM & $\mathbf{N}-\mathbf{D}$ & & & & \\
\hline S1 & $\mathrm{F}, 30$ & 0.0 & 0.0 & 0.2 & $\begin{array}{l}\text { RE: }+0.50 \mathrm{DS} \\
\mathrm{LE}:+0.25 /-0.25 \times 40\end{array}$ & Alternating, negative & Early RSOT, now 18 XOT & Mild \\
\hline NS1 & F, 19 & -0.1 & -0.1 & 0.2 & $\begin{array}{l}\text { RE: }-1.75 /-0.25 \times 180 \\
\text { LE: }+3.75 \text { DS }\end{array}$ & No suppression, 1800 & Aniso L hyper, R myop & Mild \\
\hline NS2 & M, 29 & -0.04 & -0.08 & 0.24 & $\begin{array}{l}\text { RE: }+0.50 /-0.75 \times 175 \\
\text { LE: }+2.50 /-1.50 \times 10\end{array}$ & LE partial, 500 & Aniso L hyper & Mild \\
\hline S2 & M, 36 & -0.22 & -0.16 & 0.25 & $\begin{array}{l}\text { RE: }+0.75 /-0.25 \times 130 \\
\text { LE: }+1.00 \text { DS }\end{array}$ & LE central, 3000 & 25 SOT & Mild \\
\hline S3 & M, 25 & -0.12 & -0.08 & 0.36 & $\begin{array}{l}\mathrm{RE}:+1.00 /-0.25 \times 70 \\
\mathrm{LE}:+2.25 /-0.25 \times 90\end{array}$ & LE partial, 120 & $6 \mathrm{XOT}+$ Aniso L myop & Mild \\
\hline S4 & M, 24 & -0.16 & -0.14 & 0.4 & $\begin{array}{l}\text { RE: }-0.75 /-3.50 \times 30 \\
\text { LE: }-0.25 /-3.00 \times 60\end{array}$ & LE central, negative & 15 SOT & Mild \\
\hline S5 & F, 19 & 0.02 & 0.06 & 0.48 & $\begin{array}{l}\text { RE: }-1.50 /-3.50 \times 50 \\
\text { LE: }-3.25 /-3.75 \times 120\end{array}$ & LE partial, 3000 & 15 SOT + Aniso L hyper & Mild \\
\hline NS3 & M, 19 & -0.1 & -0.08 & 0.62 & $\begin{array}{l}\text { RE: }+0.75 /-0.25 \times 40 \\
\text { LE: }+2.50 /-0.75 \times 150\end{array}$ & LE partial, 80 & Aniso L myop & Moderate \\
\hline NS4 & $\mathrm{F}, 35$ & 0.02 & 0.06 & 0.66 & $\begin{array}{l}\text { RE: }+2.50 /-4.50 \times 30 \\
\text { LE: }+0.25 /-1.00 \times 160\end{array}$ & RE partial, 250 & Aniso R myop + meridional & Moderate \\
\hline NS5 & F, 19 & 0.04 & 0.04 & 0.7 & $\begin{array}{l}\text { RE: }+0.50 /-0.75 \times 10 \\
\text { LE: }-2.50 /-0.25 \times 40\end{array}$ & LE complete, negative & Aniso L hyper & Moderate \\
\hline S6 & $\mathrm{F}, 20$ & -0.06 & -0.06 & 0.7 & $\begin{array}{l}\text { RE: }+0.50 \mathrm{DS} \\
\mathrm{LE}:-2.25 /-0.05 \times 80\end{array}$ & RE complete, negative & 30 SOT + Aniso, L hyper & Moderate \\
\hline S7 & $\mathrm{F}, 33$ & 0.04 & 0.06 & 0.72 & $\begin{array}{l}\mathrm{RE}:+4.00 /-1.00 \times 110 \\
\mathrm{LE}:+3.75 /-0.25 \times 60\end{array}$ & RE partial, negative & $20 \mathrm{SOT}$ & Moderate \\
\hline NS6 & M, 21 & -0.24 & -0.22 & 0.72 & $\begin{array}{l}\text { RE: }-0.25 /-0.25 \times 90 \\
\text { LE: }+5.50 /-0.50 \times 90\end{array}$ & LE partial, negative & Aniso L myop & Moderate \\
\hline NS7 & F, 22 & -0.08 & -0.04 & 0.82 & $\begin{array}{l}\text { RE: }-2.25 /-0.25 \times 40 \\
\text { LE: }-3.50 /-3.00 \times 160\end{array}$ & LE partial, 100 & Aniso L hyper + meridional & Moderate \\
\hline S8 & $\mathrm{F}, 20$ & -0.3 & -0.3 & 0.82 & $\begin{array}{l}\text { RE: }+0.25 \text { DS } \\
\text { LE: }+0.50 \text { DS }\end{array}$ & LE complete, negative & Late L SOT, now 25 XOT & Moderate \\
\hline s9 & $\mathrm{F}, 18$ & -0.06 & -0.06 & 0.9 & $\begin{array}{l}\text { RE: }+0.50 /-0.25 \times 140 \\
\text { LE: }+1.00 /-0.75 \times 30\end{array}$ & RE complete, negative & Early R SOT, now 60 XOT & Moderate \\
\hline NS8 & $\mathrm{F}, 21$ & -0.14 & -0.14 & 1.5 & $\begin{array}{l}\text { RE: }-1.00 \text { DS } \\
\text { LE: }+5.75 /-1.50 \times 160\end{array}$ & LE partial, negative & Aniso L myop + meridional & Severe \\
\hline S10 & M, 34 & 0.0 & 0.0 & 2.0 & $\begin{array}{l}\text { RE: }-0.25 / 4.00 \times 80 \\
\text { LE: }-0.25 / 3.75 \times 105\end{array}$ & LE complete, negative & Early L SOT, now 30 XOT & Severe \\
\hline NS9 & F, 29 & -0.2 & -0.2 & 2.0 & $\begin{array}{l}\text { RE: }+0.50 \text { DS } \\
\text { LE: }+7.25 /-1.25 \times 120\end{array}$ & LE complete, negative & $\mathrm{VD}, \mathrm{L}$ cornea + iris trauma & Severe \\
\hline NS10 & M, 48 & 0.06 & 0.06 & 2.8 & $\begin{array}{l}\text { RE: }+13.5 /-0.50 \times 180 \\
\text { LE: }-0.50 /-0.25 \times 80\end{array}$ & RE complete, negative & $\mathrm{VD}, \mathrm{R}$ cornea + lens trauma & Severe \\
\hline
\end{tabular}

Patients were classified as S, strabismic or NS, nonstrabismic. Visual acuities for binocular (BO) vision, the DOM and N-D eyes are given in $\operatorname{logMAR}$ notation. Bagolini, denotes the striated glasses test and gives the region of the affected eye (L, left; R, right) showing suppression; partial, indicates that one of the lines could only be just perceived; complete, indicates monofixation. The best crossed stereoacuity threshold (Xed SA) is given in arc sec: failure on the Titmus fly test (equivalent to a threshold of $\sim 3000$ arc sec) is recorded as negative; SOT, esotropia; XOT, exotropia; Aniso, anisometropia; VD, visual deprivation.

information available before moving, whereas later measures (e.g., the LVP, GAT, GOC, and R-GC) are expressions of the proficiency with which "online" visual feedback is used. ${ }^{20-28}$

To further probe performance accuracy, profiles of the wrist velocity and spatial trajectory and of the grip aperture obtained from each trial were examined for "errors" occurring in the later stages of the movement (see Fig. 1). Reaching errors were defined as additional movements in the velocity (e.g., Fig. 1B) or trajectory (not shown) profiles just before OC. Grasping errors were defined as additional movements in these two profiles occurring after OC and from the grip aperture profile as additional opening and closing of the digits just before or after contact (e.g., Fig. 1D), as poorly scaled (i.e., very wide) grips at $\mathrm{OC}$, and as excessively prolonged contacts with the object during the manipulation phase before lifting it. ${ }^{27}$

The overall means $( \pm \mathrm{SE})$ were calculated for each kinematic index for each participant along with the total number of reaching and grasping errors they committed across the 48 trials performed under each of the three viewing conditions. These data were entered into Huynh-Feldt-adjusted, repeated-measures ANOVA to identify within- and between-group (i.e., control subjects versus patients) effects of viewing condition, with separate analyses of depth of amblyopia (none, mild, moderate, or severe) and type of amblyopia (strabismic or nonstrabismic) as further between-subject factors. Planned least-significant difference post hoc tests were applied to identify the source(s) of any between-subjects effects. All tests of significance were based on an $\alpha$ level of 0.05 .

\section{Results}

\section{Binocular Advantages in Normal and Amblyopic Adults}

The mean data obtained for the 13 kinematic measures ( \pm the average SE) and for total reaching and grasping errors under each viewing condition in the two subject groups are shown in Table 3. Viewing condition significantly affected most aspects of performance in the normal participants, the exceptions being the reaction time (RT) and some early parameters of the 
TABLE 2. Kinematics: Abbreviations and Definitions

Parameter (Abbreviation)

Landmarks

Movement onset (MO)

Movement end (ME)

Object contact (OC)

General kinematics

Reaction time (RT)

Movement time (MT)

Reach kinematics

Peak velocity (PV)

Reach duration (RD)

Time to peak deceleration (ttPD)

Low-velocity phase (LVP)

Grasp kinematics

Time to peak grip aperture (ttPG)

Grip closure time (GCT)

Grip application time (GAT)

Peak grip aperture (PGA)

Distance from peak grip (dPG)

Grip at contact (GOC)

Coupling

Coordination between the reach and the grasp (R-GC)
Definition

Time between lens opening and wrist velocity initially exceeding $50 \mathrm{~mm} / \mathrm{s}$ Time from movement onset to target displacement exceeding $10 \mathrm{~mm}$

Moment when target displacement first exceeds $1 \mathrm{~mm}$

Movement onset time (planning + initiation)

Time from movement onset to movement end (execution)

Maximum wrist velocity (before object contact)

Time from movement onset to initial object contact

Time from movement onset to peak wrist deceleration (before object contact)

Time from peak deceleration to initial object contact

Time from movement onset to maximum grip aperture at pre-shaping

Time from maximum grip aperture to initial object contact

Time from object contact to movement end

Maximum aperture between thumb and finger (before object contact)

Distance of the wrist from the centre of the target at maximum grip aperture

Aperture between thumb and finger at initial object contact

Time between initial object contact and the minimum wrist velocity (after peak deceleration) reach (PV, ttPD) and grasp (ttPG) that are programmed in advance of the movement. Post hoc tests demonstrated that all the significant effects resulted from enhanced binocular performance, with no reliable differences between the two monocular conditions. Major binocular advantages compared with the DOM and N-D eyes alone (all $P<0.005$ ) included; faster overall movement execution times (MT) of $\sim 100 \mathrm{~ms}$, which was due to the combined effects of a shorter reaching end phase (i.e., LVP) and accompanying grip closure time (of $\sim 50-60 \mathrm{~ms}$ ), along with a subsequently reduced (by $\sim 30-40$ ms) grip application time; more accurate (i.e., smaller) grip sizes at hand preshaping (PGA) and initial object contact (GOC); and improved terminal reach-grasp synchrony (R-GC). The average SE is an indicator of the trial-to-trial variability of each kinematic measure across subjects. ANOVA revealed that the normal participants moved with greater consistency when
A

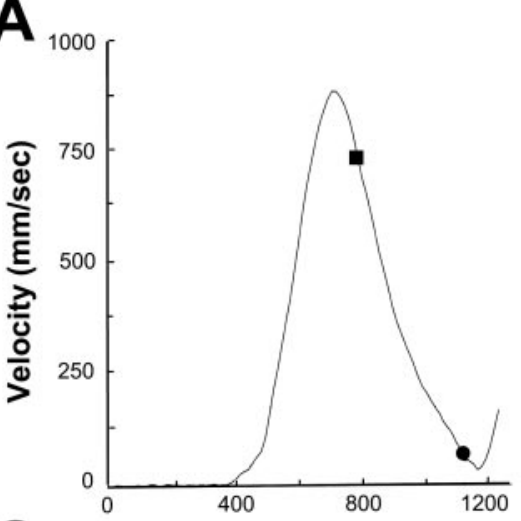

C

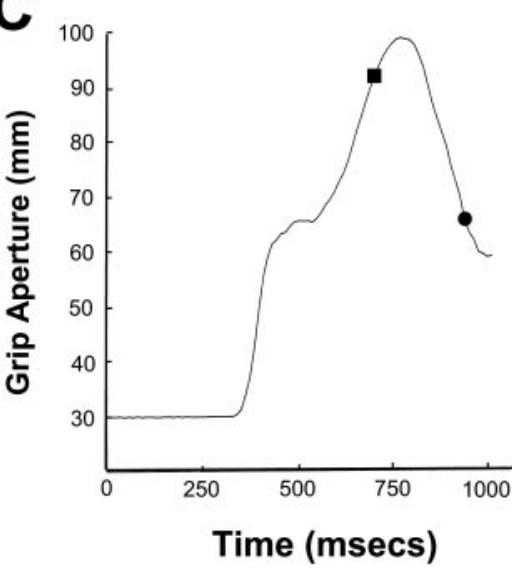

B

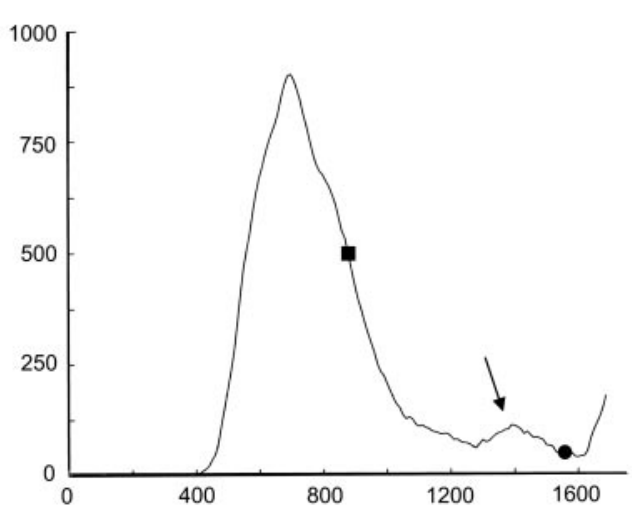

D

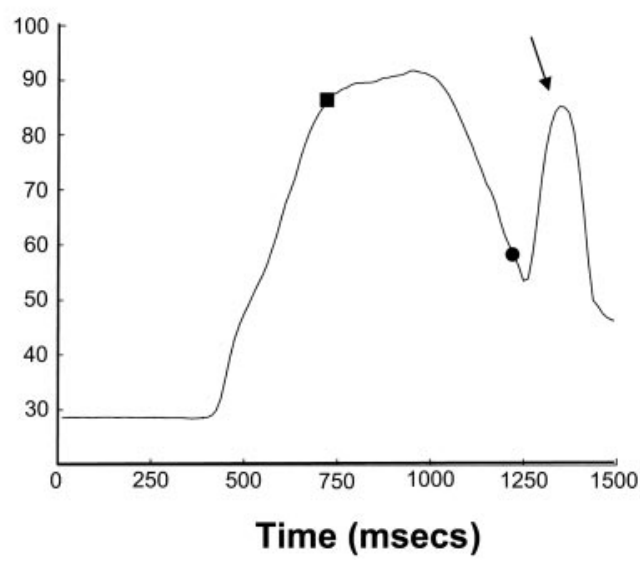

FIGURE 1. Movement profiles obtained from $(\mathbf{A}, \mathbf{C})$ binocular and $(\mathbf{B}$, D) amblyopic eye prehension trials in one of the patients with moderate VA loss. Lens opening occurred at time 0 $\mathrm{ms}$, with the moments of peak deceleration (PD) in the reach (-) and initial contact (-) with the object indicated. (A) Velocity profile of a well-executed reach, consisting of near-equal periods of acceleration and deceleration, with initial object contact (OC) occurring just before the point of minimum terminal reach velocity, followed by a brief reacceleration as the target was grasped and picked up. (B) A reaching error (arrow), in which there was an additional acceleration/deceleration (peak) in the velocity profile just before $\mathrm{OC}$, indicating a correction in the final approach to the target. (C) The grip aperture profile of a well-executed grasp, with the digits opening maximally at preshaping near the object, then rapidly closing to contact, secure, and lift the object. (D) A grasping error (arrow), in which there was an additional opening/closing of the grip just after OC, indicating a rather clumsy initial application of the grasp. 
TABLE 3. Effects of Viewing Condition on Mean Reaching and Grasping Performance in Normal and Amblyopic Adults

\begin{tabular}{|c|c|c|c|c|c|c|c|c|}
\hline \multirow[b]{2}{*}{ Parameter } & \multicolumn{4}{|c|}{ Normal } & \multicolumn{4}{|c|}{ Amblyope } \\
\hline & Binocular & $\begin{array}{c}\text { DOM } \\
\text { Eye }\end{array}$ & N-D Eye & $F_{(2,38)}$ Statistic & Binocular & $\begin{array}{c}\text { DOM } \\
\text { Eye }\end{array}$ & N-D Eye & $\mathbf{F}_{(2,38)}$ Statistic \\
\hline $\mathrm{RT}, \mathrm{ms}$ & $480 \pm 20$ & $500 \pm 23$ & $501 \pm 24$ & $2.2, P=0.1(\mathrm{NS})$ & $517 \pm 23$ & $523 \pm 23$ & $578 \pm 28$ & $7.4, P=0.01$ \\
\hline MT, ms & $796 \pm 25$ & $891 \pm 32$ & $905 \pm 32$ & $16.8, P<0.001$ & $921 \pm 34$ & $952 \pm 39$ & $1063 \pm 46$ & $34.8, P<0.001$ \\
\hline \multicolumn{9}{|l|}{ Reaching } \\
\hline $\mathrm{PV}, \mathrm{mm} / \mathrm{s}$ & $735 \pm 53$ & $705 \pm 50$ & $700 \pm 50$ & $1.0, P=0.3(\mathrm{NS})$ & $740 \pm 48$ & $739 \pm 48$ & $702 \pm 48$ & $8.3, P=0.007$ \\
\hline $\mathrm{RD}, \mathrm{ms}$ & $674 \pm 24$ & $734 \pm 29$ & $747 \pm 28$ & $8.0, P=0.007$ & $773 \pm 32$ & $791 \pm 34$ & $875 \pm 42$ & $24.6, P<0.001$ \\
\hline $\mathrm{ttPD}, \mathrm{ms}$ & $461 \pm 21$ & $457 \pm 22$ & $458 \pm 22$ & $0.0, P=0.9(\mathrm{NS})$ & $479 \pm 21$ & $477 \pm 21$ & $490 \pm 27$ & $2.2, P=0.1(\mathrm{NS})$ \\
\hline $\mathrm{LVP}, \mathrm{ms}$ & $231 \pm 28$ & $277 \pm 32$ & $289 \pm 31$ & $10.5, P=0.001$ & $294 \pm 30$ & $313 \pm 33$ & $382 \pm 42$ & $18.0, P<0.001$ \\
\hline Errors & 3.7 & 11.3 & 11.9 & $35.2, P<0.001$ & 6.9 & 10.5 & 19.1 & $29.4, P<0.001$ \\
\hline \multicolumn{9}{|l|}{ Grasping } \\
\hline PGA, mm & $77 \pm 2$ & $82 \pm 2$ & $81 \pm 2$ & $9.3, P=0.005$ & $77 \pm 2$ & $78 \pm 2$ & $83 \pm 2$ & $31.1, P<0.001$ \\
\hline $\mathrm{dPG}, \mathrm{mm}$ & $59 \pm 4$ & $69 \pm 5$ & $66 \pm 5$ & $4.8, P=0.04$ & $55 \pm 3$ & $57 \pm 3$ & $63 \pm 4$ & $4.8, P=0.04$ \\
\hline GOC, mm & $44 \pm 3$ & $47 \pm 3$ & $47 \pm 3$ & $16.9, P<0.001$ & $45 \pm 3$ & $45 \pm 3$ & $48 \pm 4$ & $5.5, P=0.018$ \\
\hline $\mathrm{ttPG}, \mathrm{ms}$ & $473 \pm 22$ & $484 \pm 23$ & $494 \pm 23$ & $0.7, P=0.4(\mathrm{NS})$ & $534 \pm 28$ & $535 \pm 26$ & $560 \pm 30$ & $8.5, P=0.005$ \\
\hline $\mathrm{GCT}, \mathrm{ms}$ & $201 \pm 16$ & $250 \pm 21$ & $253 \pm 19$ & $17.7, P<0.001$ & $240 \pm 18$ & $256 \pm 20$ & $312 \pm 29$ & $16.9, P<0.001$ \\
\hline GAT, ms & $121 \pm 12$ & $157 \pm 17$ & $158 \pm 17$ & $31.4, P<0.001$ & $148 \pm 15$ & $161 \pm 18$ & $188 \pm 23$ & $21.5, P<0.001$ \\
\hline Errors & 8.7 & 20.2 & 20.1 & $34.2, P<0.001$ & 17.7 & 22.6 & 39.4 & $28.3, P<0.001$ \\
\hline \multicolumn{9}{|l|}{ Coupling } \\
\hline R-GC, ms & $39 \pm 11$ & $70 \pm 15$ & $74 \pm 15$ & $35.3, P<0.001$ & $47 \pm 13$ & $59 \pm 16$ & $77 \pm 21$ & $12.1, P<0.001$ \\
\hline
\end{tabular}

Data are expressed as the mean \pm average SE.

using two eyes compared with either eye alone, especially for those performance indicators (e.g., MT, LVP, PGA, GAT, and R-GC) showing the greatest binocular advantages $\left(\mathrm{F}_{(2,38)}>13\right.$; $P \leq 0.001$, for all these comparisons). In accordance with this reduced variability, the normal subjects also made far fewer $(\times 0.33-0.5)$ errors during the terminal reach and the grasp when using binocular vision (see Table 3 ).

Viewing condition also affected most aspects of the amblyopes' prehension-the time to peak deceleration being the one exception-with the patients exhibiting a similar overall pattern of binocular advantage (e.g., shorter MT, LVP, GCT, GAT, improved R-GC, less variability, fewer errors) as the control subjects. Post hoc comparisons revealed that differences between binocular vision and the DOM eye were less marked on all these indices than for the normal subjects, with significantly poorer N-D eye performance relative to the other two viewing conditions (all $P<0.003$ ) being the major source of the effects (Table 2). This was particularly true of the grip size at object contact (GOC), for which the wider aperture when using the amblyopic eye was the only source of the difference. Indeed, the patients exhibited additional, if rather minor, deficits related to movement planning (slower RT, PV, and $t$ tPG) that were only apparent when they used the N-D eye, and which were not seen in the control group. In summary, although the normal subjects were much better binocularly in executing the reach and grasp, this superiority was most evident in the amblyopes relative to the N-D eye, impairments on which extended to the programming stage of the movements (see Fig. 1).

\section{Depth of Amblyopia}

Direct comparisons between the control and patient groups revealed that the amblyopes performed worse on several key measures of movement dynamics when using binocular vision, as well as under N-D eye conditions, the deficits in which covaried with existing VA loss. By contrast, there were few between-group differences in more spatial aspects of performance under these viewing conditions and in their DOM eye behavior (but see later description). Figure 2 illustrates the relationship between viewing condition and depth of amblyopia for one of the affected measures: the average movement execution time (MT, depth effect, $\mathrm{F}_{(3,36)}=4.1, P<0.015$ ). There were no differences between the normal subjects and those with mild amblyopia, but MT was significantly prolonged in both the moderate and severe amblyopes when viewing binocularly (by $\sim 125$ and $210 \mathrm{~ms}$, respectively; $P<0.025$ ) or with the N-D eye (by $\sim 180$ and $340 \mathrm{~ms}$, respectively; $P<$ 0.01). Plotting the average MT against the logMAR VA of the amblyopic eye in each patient revealed a positive correlation $\left(R^{2}=0.23\right)$ between the two for this eye, but not for binocular $\left(R^{2}=0.07\right)$ or DOM eye $\left(R^{2}=0.05\right)$ performance. An identical pattern of results (all depth effects, $\mathrm{F}_{(3,36)} \geq 2.8 P<0.05$ ), with similar correlations between N-D eye impairment and VA loss $\left(R^{2}=0.19-0.21\right)$, was obtained for two temporal parameters of the reach-its overall duration (RD) and LVP-and for the grip closure and application times. That is, the relatively extended movement times in the moderate and severe amblyopes under binocular and N-D eye conditions arose mainly because they prolonged their final approach to the object (increased

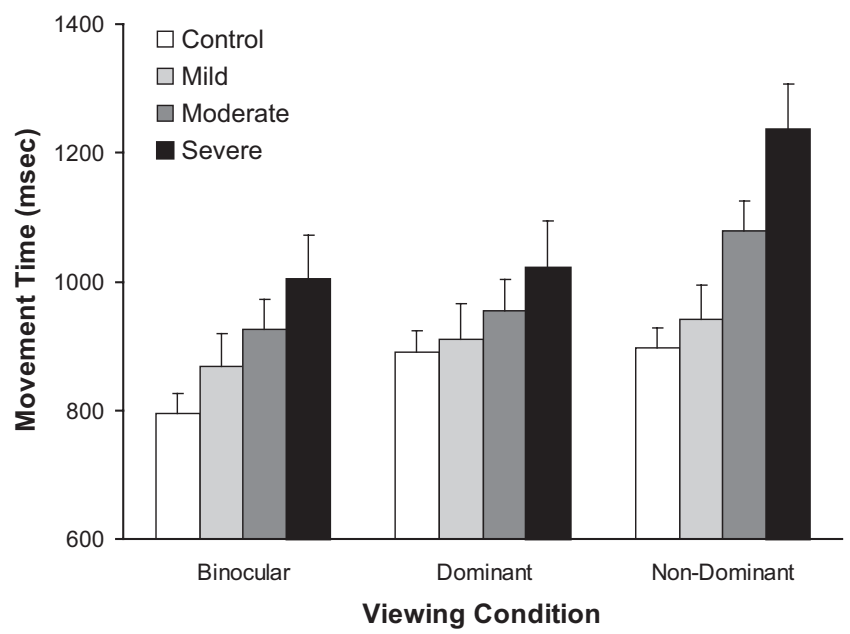

Figure 2. Average movement execution times (MT) as a function of amblyopia depth and viewing condition. Error bars, SEM. 

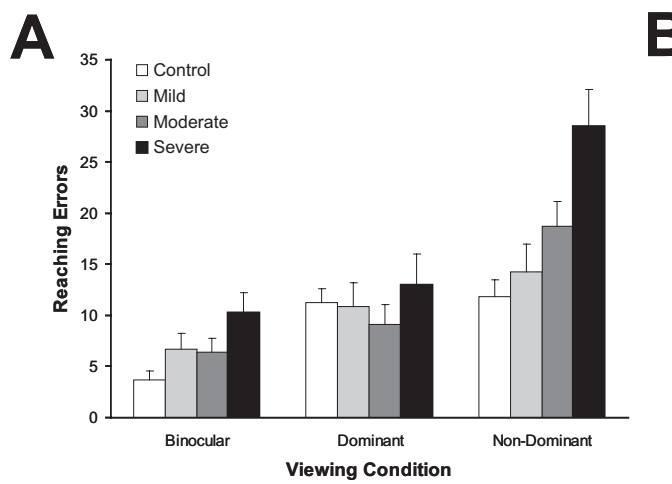

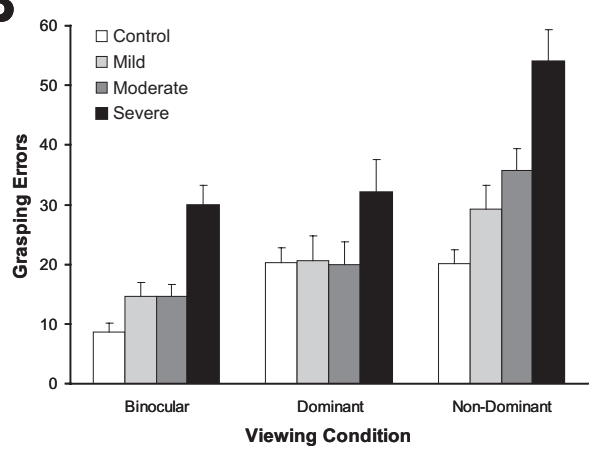

Figure 3. The average number of errors made during the (A) terminal reach and (B) the grasp as a function of amblyopia depth and viewing condition. Error bars, SEM.
LVP, GCT) and time in contact with it while securing their grip (increased GAT).

Depth of amblyopia also influenced the mean number of reaching $\left(\mathrm{F}_{(3,36)}=3.3, P<0.05\right)$ and grasping $\left(\mathrm{F}_{(3,36)}=12.9\right.$, $P<0.0001$ ) errors. For reaching errors (Fig. 3A), ANOVA showed that only the severe amblyopes were impaired relative to the control subjects under binocular conditions $(P=0.006)$, whereas those with moderate $(P<0.025)$ or severe $(P<$ $0.0001)$ VA losses were more $(\times 1.5-2.5)$ error prone when using the amblyopic eye $\left(R^{2}=0.17\right)$. In all these cases, the increases were mainly due to the patients' making more adjustments in the velocity of the reach during its LVP (e.g., Fig. 1B), rather than to alterations in its spatial path. Grasping deficits showed a different pattern (Fig. 3B). All three patient groups produced significantly more errors when using binocular vision $(\times 1.6-3.5)$ and their affected eyes $(\times 1.5-3.0)$, compared with the equivalent performance of the normal participants, and these increases mainly resulted from spatial corrections in which there were additional opening/closures of the digits just before or after object contact (e.g., Fig. 1D). Indeed, these increased grasping errors were the sole indicator of significant prehension deficits among the mild amblyopes ( $P<0.05$ for both comparisons), and this contributed to relatively strong correlations between the number of errors committed and VA loss for both binocular $\left(R^{2}=0.50\right)$ and N-D eye $\left(R^{2}=0.36\right)$ performance.

Further analyses revealed important interactions between viewing condition and subject group affecting the parameters RT, R-GC, and dPG. For the reaction time, this (view $\times$ depth) interaction $\left(\mathrm{F}_{(6,72)}=4.8, P<0.005\right)$ resulted from the severe amblyopes always taking substantially $(100-400 \mathrm{~ms})$ longer to initiate their movements than the other subjects. For R-GC it was due to the moderate and severe amblyopes contacting the object slightly earlier (by $\sim 20 \mathrm{~ms}$ ) than normal when using binocular vision (view $\times$ depth interaction, $\mathrm{F}_{(6,72)}=2.8, P<$ $0.02)$. The interaction for distance of peak grip from the target $\left(\mathrm{dPG}\right.$, view $\times$ depth, $\left.\mathrm{F}_{(6,72)}=2.5, P<0.025\right)$ was more unusual and is shown in Figure 4 . It can be seen that the mildly amblyopic subjects opened their grip to a maximum aperture closer to the target compared with the controls, and post hoc tests showed that this effect was significant for both conditions of monocular viewing (DOM eye, $P=0.014$; N-D eye, $P=$ 0.045). Moreover, dPG was also smaller across all patients under DOM eye conditions (group effect, $\mathrm{F}_{(1,38)}=7.9, P<$ 0.01 ), although these effects did not achieve significance on testing by amblyopia depth. Because opening the hand at preshaping close to the target (i.e., reduced dPG) is an element of the more confident binocular versus monocular performance in control subjects (see Table 3), these findings imply that some amblyopes are superior in this regard compared with adults with normal vision.
There was just one other indication of enhanced DOM eye performance in the patients, as a group, again related to grip accuracy at preshaping. A repeated finding in normal adults is that most reach parameters (e.g., PV, RD) increase linearly with increasing target distance, whereas others related to the grasp (e.g., PGA, GOC) increase with increasing object size. ${ }^{20,27,28}$ Figure 5 illustrates these relationships for the mean peak reaching velocity to the near-far targets and for the mean peak grip aperture for the small-large objects in the control subjects and patients under each viewing condition. The kinematics were very strongly affected by these object properties with similar scaling exhibited by both subject groups (PV, distance effect, $\mathrm{F}_{(3,36)}>500, P<0.0001$; PGA, size effect, $\mathrm{F}_{(3,36)}>400, P<$ $0.0001)$. For example, the doubling of target distance from 200 (near) to 400 (far) $\mathrm{mm}$ from the starting position resulted in a near doubling (from $\sim 500$ to $900 \mathrm{~mm} / \mathrm{s}$ ) in PV, regardless of viewing condition (Fig. 5A). Reaches were slightly faster (by $\sim 30 \mathrm{~mm} / \mathrm{s}$ ) to both distances under DOM eye conditions in the amblyopes compared with the control subjects, but this difference was not significant (eye $\times$ distance $\times$ group interaction, $P=0.2$ ). There was, however, a significant three-way (eye $\times$ size $\times$ group $)$ interaction for PGA $(P=0.002)$. Figure $5 \mathrm{~B}$ shows that this was mainly due to a difference in DOM eye performance. Specifically, the normal adults opened their grip much wider compared with binocular viewing-particularly for the smaller object-but this occurred to a much lesser extent in the patients, indicating more confident (and better) grip scaling when programming the grasp via the DOM eye.

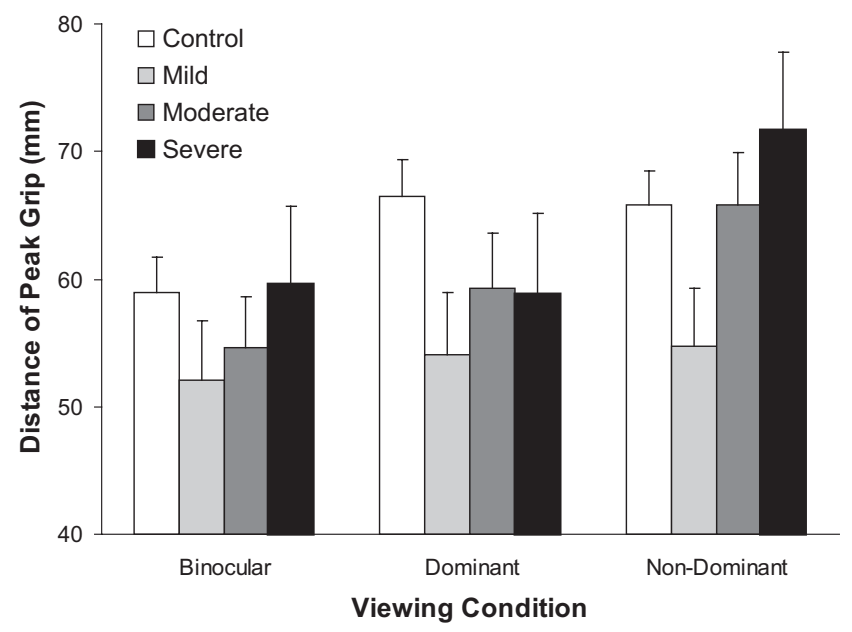

FiguRE 4. The average distance of the peak grip aperture from the target object (dPG) as a function of amblyopia depth and viewing condition. Error bars, SEM. 

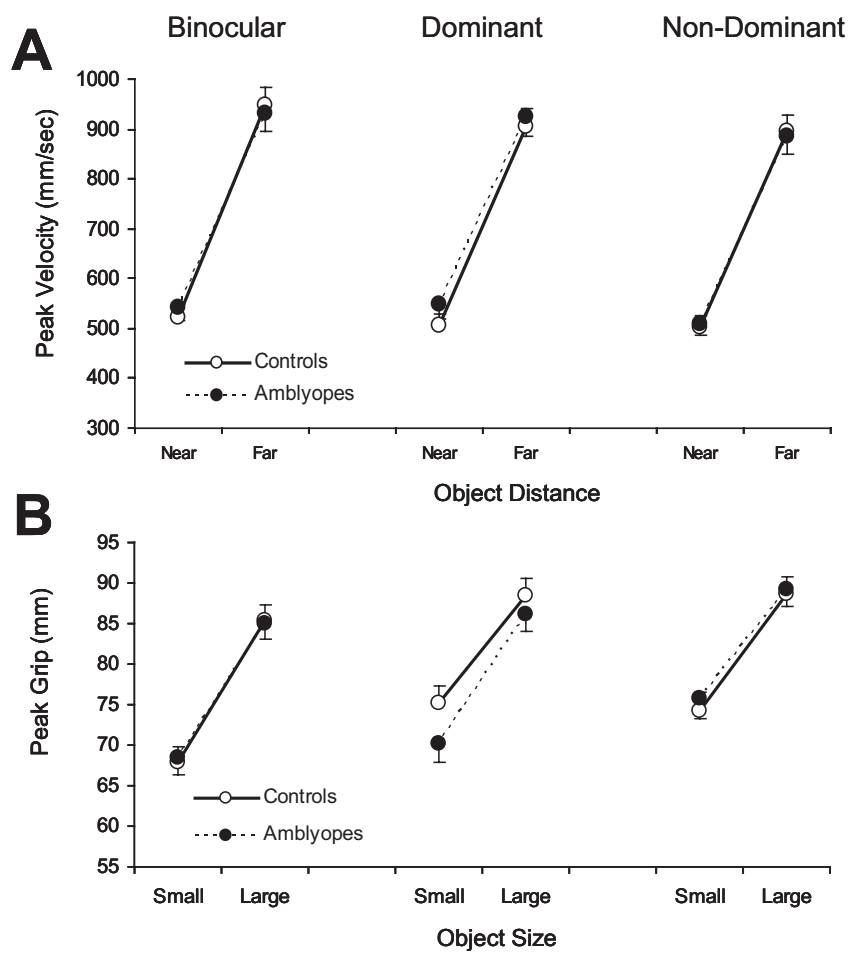

FIGURE 5. Scaling of (A) mean peak reaching velocity (PV) with target distance and of (B) mean peak grip aperture (PGA) with object size, as functions of viewing condition in the normal $(O)$ and amblyopic $(O)$ subjects. Error bars, SEM.

\section{SA Loss}

An obvious question was whether the deficits observed in the amblyopes when they performed binocularly correlated with their existing SA losses. Univariate analysis employing normal, reduced $(n=7)$, and negative $(n=13)$ SA (see Table 1$)$ as between-subject factors revealed that several measures of binocular movement timing-the mean MT, RD, and LVP (Fig. 6A)-along with the number of grasping errors (Fig. 6C) were significantly increased and by very similar extents, in both subsets of patients compared with the control subjects, with reaching errors (Fig. 6B) and GAT also increased among the patients with undetectable SA thresholds. That is, the amblyopes with residual or coarse stereopsis showed degrees of impairment similar to that of those with negative SA on some key indices of online control, while being unaffected on others.

\section{Type of Amblyopia}

Another issue was whether prehension deficits were more marked in the strabismic versus nonstrabismic patients. Analyses of the movement kinematics and error rates by type of amblyopia demonstrated no significant differences nor any reliable (view $\times$ type) interactions. Type of amblyopia also had no effect on reach velocity or grip aperture scaling. The overall VA in the affected eye of our strabismic subjects $($ mean $=0.66)$ was somewhat less than that of the nonstrabismics (mean $=$ 1.03), a difference that was not statistically significant (twotailed, unpaired $t$-test, $P>0.25$ ). These comparisons were thus considered legitimate, especially as their average binocular $(\sim 0.08)$ and DOM eye $(\sim 0.07)$ logMAR acuities were nearly identical. However, because depth of amblyopia was such an important determinant of performance, we repeated the comparisons for just the subset of 10 patients with intermediate VA losses (ranging from 0.48-0.9; see Table 1), within which there were equal distributions by amblyopia type and severity (mean N-D eye acuities, $\sim 0.7$ ). The results showed that the strabismics tended to reach more cautiously (with reduced PV and longer LVP) than the anisometropes under N-D eye conditions, but these differences were not reliable (both $P<0.1$ ). Finally, strabismics can have much greater spatial deficits in the temporal field (nasal retina) than in the nasal field (temporal retina) of the deviating eye. ${ }^{7,39}$ Our goal objects were initially placed $10^{\circ}$ to one side of the midline. Whether the target was likely to have fallen within the temporal or nasal field of the amblyopic eye at presentation, however, had no effect on performance in any of the patients groups or subsets.

\section{Discussion}

This is the first study to quantify objectively the kinematics of reaching and grasping behavior in amblyopic subjects compared with normally sighted control subjects. The main findings relating to the patients were that (1) the planned components of their movements were relatively unaffected by their vision losses, whereas (2) their online movement control exhibited multiple deficits under both amblyopic eye and binocular viewing conditions, several of which covaried with their existing depth of amblyopia, and (3) better-than-normal performance when using their DOM (good) eye was restricted to the programming of grip posture at hand preshaping.

We would emphasize that the comparative data obtained from the normal adults conformed to recent findings from our own ${ }^{27}$ and other laboratories. ${ }^{24-26}$ In particular, it has been consistently found that the initial kinematics of the reach, up to the moment of peak deceleration and including velocity scaling to target distance, can be programmed equally well from binocular or monocular assessments of the target's spatial location. Most other aspects of prehension, by contrast, are planned and executed more proficiently using two eyes, suggesting that the enhanced 3-D cues to object size, shape and relative depth provided by binocular stereopsis are particularly advantageous for grip programming/size scaling and for supplying feedback about the movement in progress. ${ }^{24-29}$ It was these aspects of performance that were most affected (either positively or negatively) in the amblyopes. We would also
Figure 6. Average (A) low-velocity phase (LVP) durations, and number of (B) reaching and (C) grasping errors during binocular prehension as a function of stereoacuity loss. Error bars, SEM. ${ }^{*} P<0.05 ;{ }^{* *} P=0.001$.

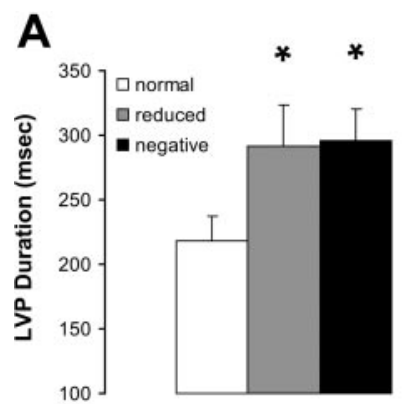

B

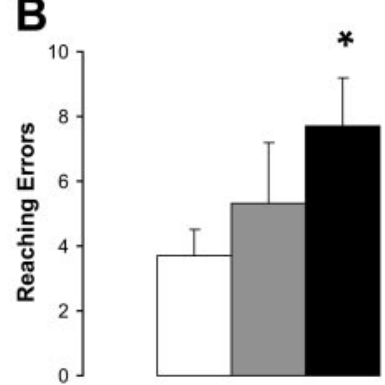

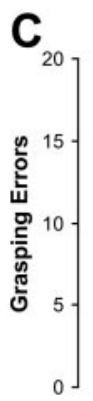

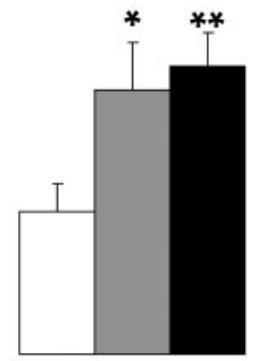


emphasize that many of the deficits in these subjects were subtle and not apparent on casual inspection during the prehension experiments, especially for those with mild amblyopia. But performance deficits under binocular and N-D eye conditions were more overt in the severe amblyopes-and in some with moderate VA loss (e.g., NS5, S7)-who were noticeably slow to react to the initial target presentation and to move toward it and who tended to apply a rather clumsy grasp.

Formal analyses by depth of amblyopia confirmed these impressions and identified the specific deficits in N-D eye performance that were related to its VA loss. In particular, they showed that while temporal features-most notably the durations of the terminal reach (LVP) and the grasp (GCT, GAT)deteriorated progressively with increasing amblyopia depth, spatial aspects of the kinematics (e.g., grip size at contact) did not. This suggests a classic speed-accuracy tradeoff: with worsening amblyopia, the patients proceeded more slowly toward the target to improve the likelihood of successfully grasping it. In this context, the increasing number of adjustments they made in terminal reach velocity (e.g., Fig. 1B) and to the grip just before contact may represent further attempts to increase end-point accuracy. That this was not always achieved, however, was made manifest by the increasing need, with worsening VA loss, to alter the grip when trying to secure the object after contact (e.g., Figs. 1D, 3B).

Depth of amblyopia also generally affected the same aspects of binocular prehension control, with that of the moderate-tosevere amblyopes increasingly resembling use of the DOM eye alone. We have examined the binocular performance of normal adults with artificial moderate-to-severe unilateral "amblyopia" induced with blurring (plus) lenses (Melmoth et al., manuscript in preparation). These subjects exhibited movement timing and grasping deficits similar to those of the real amblyopes in the present study, indicating that these effects are not secondary adaptations to long-standing visual impairment, as they can be replicated by instantaneous vision losses. This implies that treatments which restore VA to increasingly normal levels should provide immediate benefits for eye-hand coordination under natural everyday conditions (i.e., with both eyes open). But we also found that our patients' binocular performance was affected by their SA losses (Fig. 6). Some of these correlated with their degree of stereodeficiency (e.g., reaching errors), whereas others (e.g., the reach LVP duration and grasping errors) were similarly affected in the absence of normal (fine) disparity sensitivity. A confounding factor is that the reductions in VA and SA in each patient was generally correlated, as typically occurs in this disorder, ${ }^{2,40-42}$ so the relative contribution of each to the deficits in binocular control are hard to disentangle. Work in progress, ${ }^{43}$ in which we are examining the prehension of cured amblyopes, with good VA in each eye, but reduced or undetectable SA, indicates that loss of disparity sensitivity is key, since these subjects exhibit similar deficits in controlling their final approach to the target and in grasping it as the patients of the present study. This suggests that the specific objective of amblyopia therapy for improving prehension skills should be the recovery of binocularityrather than just N-D eye acuity-perhaps via greater use of modalities such as pharmacological "penalization" and refractive adaptation that allow the two eyes to work together during the treatment episodes. This recommendation is supported by recent evidence that deficits in fine visuomotor control on a binocular bead-threading task in young children with unilateral vision losses of different severities are more closely related to their accompanying reductions in SA. ${ }^{44}$

It is appealing to believe that adult amblyopes with years of experience in relying predominantly on their DOM eye for their daily requirements should learn to perform at a level equivalent to that of normal binocular vision. At the very least, it seems obvious that they will move faster and more accurately and make fewer mistakes when using this eye alone for prehension, compared with normal adults forced to do so temporarily. Our data, however, negate these notions. In fact, the only evidence we uncovered of superior DOM eye performance in the patients was that they programmed their PGA more proficiently than normal-nearer to the target and with better scaling for object size (Figs. 4, 5B)-and without adverse effects on their subsequent grasp. This suggests that amblyopes are better able to exploit monocular cues to the actual distance and size of objects with which they interact, although without fully compensating for the normal advantages of binocular vision. One cue of particular relevance could be familiar size. ${ }^{45}$ Because our subjects manipulated the objects at the end of each movement, veridical information about their real physical size and shape would have been obtained from tactile feedback. This knowledge may then have been used, on subsequent trials, to calibrate the visual angle subtended by the presented target on the retina, and so more accurately judge its size-distance relations for programming the grasp. This may also explain why their initial reach programming (e.g., ttPD, PV-distance scaling) was similar to the controls, despite their loss of spatial resolution in the N-D eye. The fact that movement planning is more amenable to such perceptual data gathered "off-line" from past experience compared with the immediate feedback demanded for online control ${ }^{46}$ is consistent with these suggestions, as is the fact that their subsequent execution of the reach and grasp was no better than normal under DOM eye conditions.

We anticipated that the underlying cause of the amblyopia might correlate with some of our patients' N-D eye deficits, especially for reaching behavior. This was partly because systematic nasal- or temporalward errors on a simple pointing task have been reported in some strabismic, but not anisometropic, amblyopes when using the affected eye. ${ }^{47}$ The pointing errors were rather variable, but tended to be largest for targets in the central $10^{\circ}$ of visual field. Because several of the strabismics exhibiting these errors had previously been shown to have nasotemporally distorted perception in central vision, ${ }^{9}$ the authors ${ }^{47}$ argued that the two phenomena were probably related and caused by disordered visuospatial mapping at high (e.g., posterior parietal) cortical levels. We presented the targets in central vision and studied strabismics with a history (e.g., change in squint direction, subjects S1, S8, S9) identified by Fronius and Sireteanu ${ }^{47}$ as favoring the occurrence of mispointing, and one subject (S2) known to have anomalous space perception. Of interest, this subject misreached on several trials, with spatial trajectories that deviated from the target location, but this did not generally occur in our strabismics, and there were only hints of other selective reaching deficits in this patient subgroup. Our findings are more compatible with altered processing at both low and high levels of the amblyope's visual cortex. The deficits, while showing some semblance to those occurring after posterior parietal damage, ${ }^{20}$ could result from downstream effects of VA and SA dysfunction in the striate cortex,${ }^{10-12}$ whereas the compensations in movement planning must clearly involve areas beyond V1. Neuroimaging may shed light on this preliminary conclusion.

Our study was an exploratory one and, as such, is subject to limitations. Some of these are common to most retrospective investigations of adult amblyopia, in that the etiology and presenting symptoms of our patient cohort were rather mixed and subdividing them by depth or type restricted the number for comparison. Our future work will place greater emphasis on eliminating these problems and will look more prospectively at potential treatment benefits for eye-hand coordination in amblyopic children. Other limitations attach to the prehension experiments. Although these were devised to 
make the task as simple and natural as possible, it was necessary to restrict the number of objects and locations used to minimize the numbers of trials. Since amblyopes may improve their performance with task familiarity, it would be interesting to examine their movements to a wider range of novel objects and positions. Viewing conditions were also randomized between trials, as is customary in these types of investigation, to reduce predictability and maintain the subjects' attention, but this does not resemble everyday experience and raises the question of whether performance would have been different were the binocular, DOM eye, and N-D eye trials to have been presented in separate blocks. We have previously examined this in a group of normal adults. ${ }^{27}$ We found that they moved faster, but with less accuracy, under blocked compared random viewing although, crucially, they also showed a very similar pattern of binocular advantage regardless of trial order. A similar effect of blocked viewing might be expected to occur in amblyopes with task predictability accentuating DOM eye compensations-for example, in programming faster reaches (see Fig. 5A). Although we intend to pursue this possibility, we would note that a previous study of adult subjects, who had undergone monocular enucleation 2 to 35 years before testing, showed that reaching performance mediated by their remaining eye was no different from that of the DOM eye of control subjects and inferior to the use of normal binocular vision. ${ }^{48}$ Other major visuomotor adaptations via the DOM eye in amblyopia would thus seem unlikely.

Amblyopia remains the commonest form of unilateral and binocular vision loss among children and younger adults, and its treatment is labor intensive and costly, as is prescreening for risk factors. This has prompted calls to justify current practice, along with a wider interest in the disabilities and quality of life associated with this condition. ${ }^{3,17}$ Our present findings add to a growing catalog of recent evidence that amblyopia and/or loss of binocularity causes a range of functional impairments, in fine visuomotor coordination, ${ }^{44}$ reading, ${ }^{18,19}$ driving, ${ }^{49}$ sports-related, ${ }^{50}$ and psychosocial ${ }^{51,52}$ skills. Taken together, these cumulative deficits have the potential to affect everyday life negatively and deserve more immediate consideration when appraising therapeutic outcomes, especially in moderate-to-severe cases of this disorder.

\section{References}

1. von Noorden GK. Binocular Vision and Ocular Motility: Theory and Management of Strabismus. 4th ed. St. Louis: Mosby; 1990.

2. Attebo K, Mitchell P, Cumming R, Smith W, Jolly N, Sparkes R. Prevalence and causes of amblyopia in an adult population. $O p h$ thalmology. 1998;105:154-159.

3. Moseley MJ, Fielder AR, eds. Amblyopia: a Multidisciplinary Approach. Woburn, MA: Butterworth-Heinemann; 2002.

4. Keech RV, Kutschke PJ. Upper age limit for the development of amblyopia. J Pediatr Ophthalmol Strabismus. 1995;32:89-93.

5. Daw NW. Critical periods and amblyopia. Arch Ophthalmol. 1998; 116:502-505.

6. Sireteanu R, Fronius M, Singer W. Binocular interaction in the peripheral visual field of humans with strabismic and anisometropic amblyopia. Vision Res. 1981;21:1065-1074.

7. Hess RF, Pointer JS. Differences in the neural basis of human amblyopia: the distribution of the anomaly across the visual field. Vision Res. 1985;25:1577-1594.

8. Levi DM, Klein SA. Vernier acuity, crowding and amblyopia. Vision Res. 1985;25:979-991.

9. Fronius M, Sireteanu R. Monocular geometry is selectively distorted in the central visual field of strabismic amblyopes. Invest Ophthalmol Vis Sci. 1985;30:2034-2044.

10. Baker FH, Grigg P, von Noorden GK. Effects of visual deprivation and strabismus on the response of neurons in the visual cortex of the monkey, including studies of striate and prestriate cortex in the normal animal. Brain Res. 1974;66:185-208.
11. Kiorpes L, Kiper DC, O'Keefe LP, Cavanaugh JR, Movshon JA. Neuronal correlates of amblyopia in the visual cortex of macaque monkeys with experimental strabismus and anisometropia. $J$ Neurosci. 1998;18:6411-6424.

12. Kiorpes L, McKee SP. Neural mechanisms underlying amblyopia. Curr Opin Neurobiol. 1999;9:480 - 486.

13. Imamura $\mathrm{K}$, Richter $\mathrm{H}$, Fischer $\mathrm{H}$, et al. Reduced activity in the extrastriate visual cortex of individuals with strabismic amblyopia. Neurosci Letts. 1997;225:173-176.

14. Barnes GR, Hess RF, Dumoulin SO, Achtman RL, Pike GB. The cortical deficit in humans with strabismic amblyopia. J Physiol (Lond). 2001:533;281-297.

15. Anderson SA. Neuroimaging in human amblyopia. Strabismus. 2006:14;21-35.

16. Sharma V, Levi DM, Klein SA. Undercounting features and missing features: evidence for a high-level deficit in strabismic amblyopia. Nat Neurosci. 2000;3:496-501.

17. Snowdon SK, Stewart-Brown S. Preschool vision screening: results of a systematic review. Report 9. York NHS Centre for Reviews and Dissemination. York, UK: University of York; 1997:1-92.

18. Simons HD, Gassler PA. Vision anomalies and reading skill: a meta-analysis of the literature. Am J Optom Physiol Opt. 1988;65: 893-904.

19. Stifter E, Burggasser G, Hirmann E, Thaler A, Radner W. Monocular and binocular reading performance in children with microstrabismic amblyopia. Br J Ophthalmol. 2005;89:1324-1329.

20. Jeannerod M. Mechanisms of visuomotor coordination: a study in normal and brain-damaged subjects. Neuropsychologica. 1986;24: 41-78.

21. Sivak B, MacKenzie CL. Integration of visual information and motor output in reaching and grasping: the contributions of peripheral and central vision. Neuropsychologica. 1990;28:1095-1116.

22. Watt SJ, Bradshaw MF, Rushton SK. Field of view affects reaching, not grasping. Exp Brain Res. 2000;135:411-416.

23. Marotta JJ, Goodale MA. The role of learned pictorial cues in the programming and control of grasping. Exp Brain Res. 1998;121: 465-470.

24. Mon-Williams M, Dijkerman HC. The use of vergence information in the programming of prehension. Exp Brain Res. 1999;128:578-582.

25. Watt SJ, Bradshaw MF. Binocular cues are important in controlling the grasp but not the reach in natural prehension movements. Neuropsychologica. 2000;38:1473-1481.

26. Loftus A, Servos P, Goodale MA, Mendarozqueta N, Mon-Williams M. When two eyes are better than one in prehension: monocular viewing and end-point variance. Exp Brain Res. 2004;158:317-327.

27. Melmoth DR, Grant S. Advantages of binocular vision for the control of reaching and grasping. Exp Brain Res. 2006;171:371388.

28. Watt SJ, Bradshaw MF. The visual control of reaching and grasping: binocular disparity and motion parallax. J Exp Psychol Hum Percept Perform. 2003;29:404-415.

29. Melmoth DR, Storoni M, Grant S. Dissociation between the use of vergence and binocular disparity information in the control of reaching and grasping movements (Abstract). Perception. 2005; 34(suppl):247.

30. von Hofsten C. Binocular convergence as a determinant of reaching behaviour in infancy. Perception. 1977;6:139-144.

31. Granud CE. Binocular vision and spatial perception in 4- and 5-month old infants. J Exp Psychol Hum Percept Perform. 1986; $12: 36-49$.

32. Kuhtz-Buschbeck JP, Stolze H, Johnk K, Boczek-Funcke A, Illert M. Development of prehension movements in children: a kinematic study. Exp Brain Res. 1998;122:424- 432.

33. Pare M, Dugas C. Developmental changes in prehension during childhood. Exp Brain Res. 1999;125:239-247.

34. Smyth MM, Peacock KA, Katamba J. The role of sight of the hand in the development of prehension in childhood. QJ Exp Psychol. 2004;57A:269-296.

35. Reed MJ, Steeves JKE, Steinbach MJ, Kraft S, Gallie B. Contrast letter thresholds in the non-affected eye of strabismic and unilateral eye enucleated subjects. Vision Res. 1996;36:3011-3018. 
36. Freeman RD, Bradley A. Monocularly deprived humans: non-deprived eye has supernormal vernier acuity. J Neurophysiol. 1980 43:1645-1653.

37. Bradley A, Freeman RD. Contrast sensitivity in anisometropic amblyopia. Invest Ophthalmol Vis Sci. 1981;21:467-476.

38. Oldfield RC. The assessment and analysis of handedness: the Edinburgh inventory. Neuropsychologica. 1971;9:97-112.

39. Sireteanu R, Fronius M. Naso-temporal asymmetries in human amblyopia: consequences of long-tern interocular suppression. Vision Res. 1981;21:1055-1063.

40. Goodwin RT, Romano PE. Stereoacuity degradation by experimental and real monocular and binocular amblyopia. Invest Ophthalmol Vis Sci. 1985;26:917-923.

41. Weakley DR. The association between nonstrabismic anisometropia, amblyopia, and subnormal binocularity. Ophthalmology. 2001;108:163-171.

42. Lee SY, Isenberg SJ. The relationship between stereopsis and visual acuity after occlusion therapy for amblyopia. Ophthalmology. 2003;110:2088-2092.

43. Grant S, Melmoth DR, Morgan MJ, Finlay AL. Deficits in the on-line control of reaching and grasping actions in adults with reduced binocular vision (Abstract). Ophthal Physiol Optics. 2005:25:465.
44. Hrisos A, Clarke MP, Kelly T, Henderson J, Wright CM. Unilateral visual impairment and neuro-developmental performance in preschool children. Br J Ophthalmol. 2006;90:836-838.

45. Marotta JJ, Goodale MA. The role familiar size in the control of grasping. J Cogn Neurosci. 2001;13:8-17.

46. Glover S. Visual illusions affect planning but not control. Trends Cogn Neurosci. 2002;6:288-292.

47. Fronius M, Sireteanu R. Pointing errors in strabismics: complex patterns of distorted visuomotor coordination. Vision Res. 1994; 34:689-707.

48. Marotta JJ, Perrot TS, Nicolle D, Servos P, Goodale MA. Adapting to grasping with one eye. Exp Brain Res. 1995;104:107-114.

49. Bauer A, Dietz K, Kolling G, Hart W, Schiefer U. The relevance of stereopsis for motorists: a pilot study. Graefes Arch Clin Exp Ophthalmol. 2001;239:400-406.

50. Lenoir M, Musch E, La Grange N. Ecological relevance of stereopsis in one-handed ball-catching. Percept Motor Skills. 1999; 89:495-508

51. Satterfield D, Keltner JL, Morrison TL. Psychosocial aspects of strabismus study. Arch Ophthalmol. 1993;111:1100-1105.

52. Packwood EA, Cruz OA, Rychwalski PJ, Keech RV. The psychosocial effects of amblyopia study. J AAPOS. 1999;3:15-17. 